\title{
Nils Reiter
}

\section{Anleitung zur Erstellung von Annotationsrichtlinien}

Zusammenfassung: In diesem Kapitel wird eine kurze Anleitung in die Entwicklung von Annotationsrichtlinien gegeben, unter der Annahme dass die Richtlinien ein Phänomen abdecken sollen, das bereits theoretisch beschrieben wurde. Ziel des Prozesses ist dann, Annotationsrichtlinien sowohl so generisch wie möglich als auch so präzise wie möglich zu gestalten. Menschliche Annotatorinnen und Annotatoren sollen auf Basis der Richtlinien sicher und zuverlässig annotieren können.

Abstract: This chapter gives a brief practical introduction into the development of annotation guidelines, for the scenario that new guidelines are created for a phenomenon or concept that has been described theoretically. In a single sentence, the goal of annotation guidelines can be formulated as: Given a theoretically described phenomenon or concept, describe it as generic as possible but as precise as necessary so that human annotators can annotate the concept or phenomenon in any text without running into problems or ambiguity issues.

\section{Einleitung}

Annotationsrichtlinien sollen ein Phänomen oder theoretisch gegebenes Konzept so generisch wie möglich, aber gleichzeitig so genau wie nötig beschreiben, damit menschliche Annotatorinnen und Annotatoren zuverlässig und intersubjektiv annotieren können. Mehrdeutigkeiten, also Stellen an denen mehrere Annotationskategorien möglich sind, sollen zumindest bemerkt werden und nicht stillschweigend untergehen. Die Erstellung der Richtlinien erfolgt dabei in einem iterativen Prozess: Sobald eine erste (Proto-)Version erstellt wurde, kann sie getestet werden. Dies erfolgt durch Anwendung, also die Annotation von Texten. Dadurch werden Unzulänglichkeiten sichtbar, die in einer nächsten Version der Richtlinien behoben werden. Diese wiederum werden getestet, was weitere Unzulänglichkeiten sichtbar macht. Diese können dann in einer weiteren Version behoben werden.

Anmerkung: Diese Anleitung wurde im Rahmen des shared tasks SANTA, Teil IV dieses Bandes, erstellt und zuerst auf https://sharedtasksinthedh.github.io/2017/10/01/howto-annotation/ publiziert. Für den Abdruck hier wurde sie übersetzt, überarbeitet und erweitert.

Nils Reiter, Institut für Maschinelle Sprachverarbeitung, Universität Stuttgart

○ Open Access. (c) 2020 Nils Reiter, publiziert von De Gruyter (c) BY-NC-ND Dieses Werk ist lizenziert unter der Creative Commons Attribution-NonCommercial-NoDerivatives 4.0 International Lizenz. https://doi.org/10.1515/9783110693973-009 


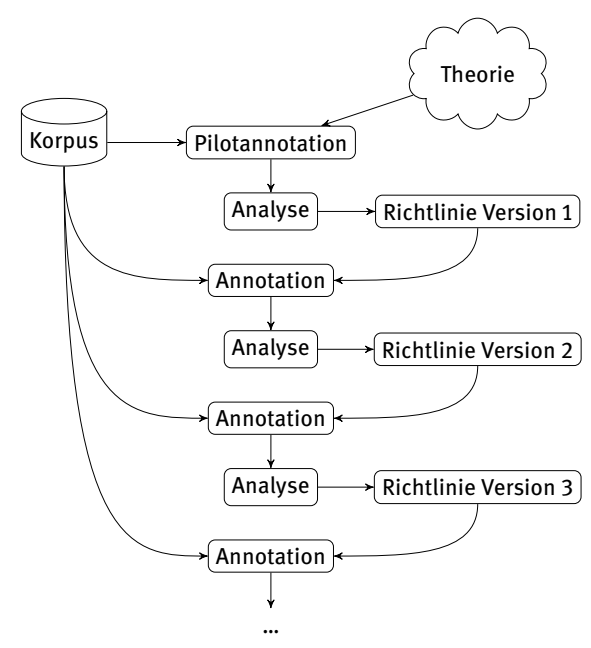

Abb. 1: Allgemeiner Arbeitsablauf. Der Prozess endet, wenn die Analyse ergibt, dass die Richtlinien hinreichend zuverlässig angewendet werden können.

Dieser Prozess ist schematisch dargestellt in Abbildung 1. Im folgenden wird genauer beschrieben, wie Richtlinien von einer Version zur nächsten weiterentwickelt werden können. Die wichtigste Idee dabei ist, dass der gleiche Text von mehreren Annotatorinnen und Annotatoren unabhängig voneinander bearbeitet wird.

Prinzipiell kann das gesamte Verfahren mit oder ohne Einsatz von Computern erfolgen. Digitale Annotationswerkzeuge erleichtern einige Arbeiten allerdings massiv, etwa den Vergleich von Annotationen verschiedener Annotatorinnen und Annotatoren. Außerdem zwingen sie die Annotatorinnen und Annotatoren dazu, sich darauf festzulegen, welche Wörter genau gemeint sind. Dies ist schwer auf Papier umzusetzen. Papier-basierte Annotationen sind dagegen zugänglicher und leichter aufzusetzen, können aber auch dazu führen, dass die Annotationen weniger exakt lokalisiert werden, oder ihrerseits nicht eindeutig sind (etwa weil Kategorien einfach am Seitenrand stehen). Für viele Arbeitsschritte, die sich an die Annotation anschließen, sind ohnehin exakte Grenzen nötig. Daher sollte man auch bei Papier-basierten Annotationen auf diese achten.

\section{Pilotannotationen}

Die erste Annotationsrunde wird am Besten von Annotatorinnen und Annotatoren durchgeführt, die mit der Theorie, gemäß der annotiert werden soll, vertraut 
sind. Die Annotation sollte gleichzeitig und unabhängig voneinander durchgeführt und insbesondere sollten ad-hoc-Absprachen zwischen den Annotatorinnen und Annotatoren vermieden werden. Eine erste Annotationsrunde kann mit minimaler Vorbereitung starten. Im Regelfall sollte es genügen, eine oder mehrere wissenschaftliche Grundlagenwerke zur Orientierung und den zu annotierenden Text oder Textabschnitt festzulegen.

Ist ein Text oder Textabschnitt annotiert, sollten die Annotationen verglichen und besprochen werden. Dass die Annotationen schriftlich fixiert sind, zwingt die Annotatorinnen und Annotatoren dazu, ihre Entscheidungen ggf. begründen zu müssen. Die Annotationsunterschiede zu diskutieren, ist ein zentraler Mechanismus, Unschärfen in den Richtlinien offenzulegen. Gerade am Anfang sind viele Parameter noch nicht festgelegt und daher Ursache von Annotationsunterschieden. In der Diskussion sollte der Fokus zunächst auf den folgenden, eher formalen Punkten liegen. Ist der Annotationsprozess weiter fortgeschritten, verschiebt sich der Fokus zu inhaltlicheren Fragen (s. u.):

- Was genau wird annotiert? Jeder Absatz, jeder Satz, jedes Wort? Nur Einheiten, die eine bestimmte Bedingung erfüllen? Hier sollte eine möglichst klare und interpretationsunabhängige Einheit gewählt werden, so dass die eigentliche Entscheidung in der Kategorisierung der Einheiten liegt.

- Was genau sind die Annotationskategorien? Wie sind sie untereinander verwandt? Schließen sie sich zum Beispiel gegenseitig aus? In manchen Fällen hilft es vielleicht, die Kategorien zu hierarchisieren (z. B. finites Verb ist eine Unterkategorie von Verb).

- Mit welchem Tool wird annotiert? Müssen tool-spezifische Festlegungen getroffen werden? Sind alle Annotatorinnen und Annotatoren mit der Benutzung vertraut? Wie können Lesezeichen oder Kommentare gesetzt werden?

Diese ersten Entscheidungen und ihre Gründe sollten dokumentiert werden. Aus dieser Dokumentation ergeben sich dann nach und nach die Annotationsrichtlinien. Annotationsrichtlinien zeichnen sich in der Praxis durch eine Vielzahl von Beispielen aus. Es ist nie zu früh, mit dem Sammeln von interessanten, schwierigen und aufschlussreichen Beispielen anzufangen. Beispiele aus ,echten' Texten sind zwar hilfreicher als ausgedachte, allerdings kann und sollte der ,Knackpunkt ' herausgearbeitet werden (z. B. indem Teile des Beispiels weggelassen werden, die für die Entscheidung nicht relevant sind). 


\section{Richtlinien verbessern}

Um Richtlinien in diesem Szenario zu verbessern, müssen zuerst die Annotationen, die mit der früheren Version der Richtlinien erstellt wurden, analysiert werden. Dabei sollte der Fokus sinnvollerweise auf den Annotationsunterschieden liegen, also den Stellen, an denen die Annotatorinnen und Annotatoren unterschiedliche Entscheidungen getroffen haben. Diese können natürlich quantifiziert werden (s. u.), aber gerade zu Beginn ist es ergiebiger mit den Annotatorinnen und Annotatoren über ihre Entscheidungen zu sprechen. Dabei können die folgenden Fragen eine Orientierung geben:

- Welche Kategorien waren besonders einfach $\mathrm{zu}$ annotieren, welche besonders schwer?

- In welchen Fällen widersprachen Entscheidungen, die aufgrund der Richtlinien getroffen wurden, der eigenen Intuition?

- In welchen Fällen konnte keine Entscheidung getroffen werden, weil mehrere Kategorien ,passten‘?

- In welchen Fällen konnte keine Entscheidung getroffen werden, weil keine Kategorie ,passte‘?

Sind die Annotatorinnen und Annotatoren eingearbeitet und trainiert, genügt womöglich ein quantitativer Blick auf die Annotationsunterschiede zur Weiterarbeit. Dafür wurden eine Reihe von Metriken unter den Stichworten inter-annotator agreement oder inter-rater reliability vorgeschlagen (cf. Artstein und Poesio 2008, für eine Übersicht). Die meisten Metriken sind eine Kombination aus tatsächlich beobachteter (observed) und erwarteter (expected) Übereinstimmung. Die beobachtete Übereinstimmung wird aus den Annotationen ermittelt, etwa als Anteil der paarweisen Übereinstimmungen für die Metrik Fleiss' $\kappa$ (Fleiss 1971). Erwartete Übereinstimmung zu messen, ist eher erklärungsbedürftig.

Im Falle von Fleiss' $\kappa$ wird mit der erwarteten Übereinstimmung gemessen, welche Übereinstimmung erzielt worden wäre, wenn alle Annotatorinnen und Annotatoren zufällige Entscheidungen getroffen hätten. Die Überlegung dahinter ist, dass auch bei rein zufälligen Entscheidungen noch ein gewisses Maß an Übereinstimmung erzielt wird - wie hoch diese ist, hängt stark von der Anzahl der Kategorien ab. Um eine realistische Einschätzung der echten Übereinstimmung zwischen den Annotatorinnen und Annotatoren zu erhalten, wird nun die zufällige Übereinstimmung mit der beobachteten verrechnet. Das Ergebnis davon ist eine Zahl, etwa im Intervall zwischen - $\infty$ und 1, wobei ein Wert von 0 ausdrückt, dass 
die Übereinstimmung der Annotatorinnen und Annotatoren nicht besser ist als die zufällige Übereinstimmung. ${ }^{1}$

Nur wenige Metriken differenzieren die Arten der Nicht-Übereinstimmung. In der Praxis ist es aber relevant, ob eine Nicht-Übereinstimmung aus Unachtsamkeit oder Missinterpretation der Definitionen erfolgt ist. Entscheidungen aus Unachtsamkeit sind leicht reparierbar und erfordern auch keine Neudefinition der Richtlinien, während Entscheidungen aus Missinterpretation der Definitionen sehr wohl Anlass geben, die Definitionen zu überdenken: Ein zusätzliches Beispiel kann diese klarer machen, oder auch eine Neuformulierung der Definition selbst. Gegebenenfalls kann auch die Klärung von Begriffen notwendig sein. Gius und Jacke (2017) unterscheiden in diesem Zusammenhang noch eine weitere Kategorie von Nicht-Übereinstimmungen, die je nach Komplexitätsgrad der Annotationsaufgabe benötigt wird: Divergierende Vorannahmen beziehen sich darauf, dass die Entscheidung für oder gegen eine Annotationskategorie von Interpretationen des Textes abhängen kann, die nicht Teil der Annotationsaufgabe sind. Es ist darüber hinaus zu erwarten, dass nicht alle Annotationsentscheidungen eindeutig sein werden. Einige Annotationsunterschiede basieren auf echten Mehrdeutigkeiten von Textstellen oder Texten und lassen sich auch nicht ausräumen.

Viele Annotationsunterschiede werden in der Praxis von Fällen verursacht, die in den Richtlinien bisher nicht abgedeckt sind. In diesem Fall muss entweder eine existierende Definition/Kategorie übertragen (und entsprechend erweitert) oder eine neue Kategorie definiert werden. Bei allen Änderungen an den Kategorien müssen deren Auswirkungen auf vergangene Annotationen mitgedacht werden. Hätte es die Annotationen verändert, wenn die Kategorie schon bestanden hätte? Teilweise muss dann ein Teil des Korpus neu annotiert (oder nicht mehr weiter verwendet) werden.

Die Diskussionen über konkrete Texte sind erfahrungsgemäß intensiv und verlaufen ungesteuert teilweise assoziativ. Eine gute Gesprächsführung und Moderation ist daher wichtig, um die verschiedenen Annotationsprobleme sauber zu trennen und auch die Entscheidungen zu dokumentieren. Nicht alle Probleme lassen sich auf einmal lösen, manchmal ist das Zurückstellen bestimmter Fragen die einzig richtige Antwort - wenn mehr Beispiele zum gleichen Phänomen diskutiert wurden und vorliegen, kann ggf. leichter eine Entscheidung getroffen werden.

Während der Prozess auf diese Weise mehrere Phasen durchläuft, passieren zwei Dinge gleichzeitig und miteinander verwoben: Sowohl die Annotationsrichtlinien, als auch die Annotatorinnen und Annotatoren werden besser, wobei ,bes-

1 Verschiedene Metriken verwenden unterschiedliche Skalen. 
ser' im letzten Fall auch heißen kann, dass sie besser antizipieren, was gewünscht wird, ohne dass es expliziert wurde. Eine höhere Übereinstimmung muss also nicht zwangsläufig bedeuten, dass die Richtlinien besser werden. Um das wirklich zu testen, bietet es sich an, gelegentlich neue Annotatorinnen und Annotatoren einzubeziehen, um deren Feedback zu bekommen. Dadurch werden auch ungeschriebene Regeln, die sich innerhalb eines Projektes entwickeln, transparent und sichtbar.

\section{Annotationsrichtlinien finalisieren}

Der in Abbildung 1 gezeigte Workflow kann theoretisch ohne Ende fortgeführt werden. Ist es das Ziel der Arbeit, theoretische Erkenntnisse über die annotierten Konzepte zu erzielen, kann die Annotation über sehr lange Zeiträume auch ein angemessenes Vorgehen sein (cf. Pagel et al. 2020, in diesem Band). Werden die Richtlinien zu dem Zweck entwickelt, möglichst viele Annotationen in einem Folgeschritt zu verwenden, muss eine Richtlinie zu einem Zeitpunkt als fertig deklariert werden. Eine Möglichkeit dazu ist, ein Mindestmaß an inter-annotator agreement zu definieren, das auf jeden Fall erreicht werden soll.

In Abbildung 2 findet sich eine Empfehlung für eine Gliederung einer Richtlinie. $\mathrm{Zu}$ beachten ist dabei, dass es sich bei Annotationsrichtlinien nicht primär um wissenschaftliche Texte handelt - die Richtlinien sollen zuvörderst von den Annotierenden verwendet werden. Dabei kann man drei unterschiedliche Verwendungsweisen unterscheiden: (i) Bei neu angefangenen Annotationsprojekten dienen die Richtlinien dazu, die zu annotierenden Einheiten und Kategorien erstmalig zu definieren. Sind die Annotierenden mit den Grundlagen hinreichend vertraut, entwickeln sich die Richtlinien weiter zu (ii) einem Nachschlagewerk, in dem vergangene Annotationsentscheidungen dokumentiert sind. Stößt eine Annotationskraft auf ein neues Beispiel, für das eine bereits getroffene Entscheidung ebenfalls angewendet werden kann, muss diese Entscheidung schnell auffindbar sein. Dies kann mit einem Index, einem großzügigen Layout oder einer Volltextsuche unterstützt werden. (iii) Ein dritter Anwendungsfall ist das Einarbeiten neuer Annotierender. Bei länger laufenden Annotationsprojekten lässt sich nicht vermeiden, dass die Annotierenden wechseln, und neue Annotierende eingelernt werden müssen. Diese werden die Richtlinien zunächst wieder von vorne lesen, aber ohne den Entwicklungsprozess miterlebt zu haben. Es ist also darauf zu achten, dass auch bei vielen kleineren Änderungen an den Richtlinien, die einleitenden Teile noch zum Kern der Richtlinien passen. Diesem Anwendungsfall ähnlich 
1. Einleitung

- Worum geht es? Was soll annotiert werden?

- Worauf basieren die Richtlinien?

- Wer hat sie wann in welchem Projekt erstellt?

- An wen richten sie sich? Welche Kenntnisse werden auf Seiten der Annotierenden vorausgesetzt?

2. Annotationseinheiten

- Was sind die zu annotierenden Einheiten (z. B. Wörter, Sätze, Absätze, ...)?

- Sind alle Einheiten zu annotieren oder nur bestimmte? Woran erkennt man sie?

3. Annotationskategorien

- Welche Kategorien werden den Einheiten zugewiesen?

- Woran erkennt man eine Kategorie?

- Welche Kategorien sind ggf. leicht zu verwechseln?

- Gibt es Abhängigkeiten zwischen den Kategorien?

4. Problematische Fälle

- Was sind schwierige Fälle?

- Wie wurde bei denen entschieden, und warum?

5. Praktische Umsetzung

- Wie genau ist das Annotationstool zu verwenden?

- Falls das Annotationstool eine eigene Anleitung bereitstellt, kann auch auf diese verwiesen werden. In dem Fall sollte aber ggf. eine ,Übersetzung' der Begriffe mitgeliefert werden.

6. Änderungsprotokoll

- Welche Stellen wurden in welcher Iteration geändert?

Abb. 2: Gliederungsempfehlung für Annotationsrichtlinien 
ist auch die Weitergabe an andere Forschende, die sich für das annotierte Korpus, die annotierten Konzepte oder die Annotationsrichtlinien als solche interessieren.

Stilistisch empfehlen wir, eher Stichpunkte und Aufzählungen als Fließtext zu verwenden. Die Nummerierung von Beispielen macht es leichter mit ihnen zu arbeiten. Wissenschaftliche Referenzen können im Sinne der Nachvollziehbarkeit angegeben werden, es sollte aber eher vermieden werden, deren Rezeption zur Voraussetzung zu machen. Für das Sammeln und Erstellen der Richtlinien hat sich in CRETA ein Wiki als geeignet erwiesen, es sollte allerdings darauf geachtet werden, dass eine Druckversion extrahierbar ist (in längeren Richtlinien ist die Orientierung und Zugänglichkeit eine Herausforderung, und im Druck fällt das vielen Menschen leichter).

\section{Beispiele für Annotationsrichtlinien}

Beispiele für Annotationsrichtlinien zum literarischen Phänomen der Erzählebene finden sich in der Cultural-Analytics-Sonderausgabe zum SANTA shared task (Gius, Reiter et al. 2019). Mit Barth (2020) und Ketschik, Murr et al. (2020) befinden sich zwei von ihnen auch in diesem Band ab Seite 423. Daneben dokumentiert der vorliegende Band auch die interdisziplinäre Entwicklung einer Annotationsrichtlinie für Entitätenreferenzen (Ketschik, Blessing et al. 2020, S. 204 ff.), sowie die Konzeptualisierung von Annotationen für Emotionen in Erzähltexten (Klinger et al. 2020, S. 238 ff.). Eine Sammlung computerlinguistischer Annotationsrichtlinien wurde von Ide und Pustejovsky (2017) publiziert.

Danksagung: Am Inhalt dieser Anleitung haben Evelyn Gius und Marcus Willand im Rahmen des SANTA-shared tasks (Willand et al. 2020, in diesem Band) mitgewirkt.

\section{Literatur}

Artstein, Ron und Massimo Poesio (2008). „Inter-Coder Agreement for Computational Linguistics“. In: Computational Linguistics 34.4, S. 555-596.

Barth, Florian (2020). „Annotation narrativer Ebenen und narrativer Akte“. In: Reflektierte Algorithmische Textanalyse. Hrsg. von Nils Reiter, Axel Pichler und Jonas Kuhn. Berlin: De Gruyter, S. 423-438.

Fleiss, Joseph L. (1971). „Measuring nominal scale agreement among many raters“. In: Psychological Bulletin 76.5, S. 420-428. 
Gius, Evelyn und Janina Jacke (2017). „The Hermeneutic Profit of Annotation: On Preventing and Fostering Disagreement in Literary Analysis“. In: International Journal of Humanities and Arts Computing 11.2, S. 233-254.

Gius, Evelyn, Nils Reiter und Marcus Willand, Hrsg. (2019). Cultural Analytics: A Shared Task for the Digital Humanities: Annotating Narrative Levels.

Ide, Nancy und James Pustejovsky, Hrsg. (2017). Handbook of Linguistic Annotation. Berlin/Heidelberg: Springer.

Ketschik, Nora, André Blessing, Sandra Murr, Maximilian Overbeck und Axel Pichler (2020). „Interdisziplinäre Annotation von Entitätenreferenzen“. In: Reflektierte Algorithmische Textanalyse. Hrsg. von Nils Reiter, Axel Pichler und Jonas Kuhn. Berlin: De Gruyter, S. 204236.

Ketschik, Nora, Sandra Murr, Benjamin Krautter und Yvonne Zimmermann (2020). „Zur Theorie von Erzählebenen und ihrer Annotation im digitalen Kontext“. In: Reflektierte Algorithmische Textanalyse. Hrsg. von Nils Reiter, Axel Pichler und Jonas Kuhn. Berlin: De Gruyter, S. 440-464.

Klinger, Roman, Evgeny Kim und Sebastian Padó (2020). „Emotion Analysis for Literary Studies“. In: Reflektierte Algorithmische Textanalyse. Hrsg. von Nils Reiter, Axel Pichler und Jonas Kuhn. Berlin: De Gruyter, S. 238-268.

Pagel, Janis, Nils Reiter, Ina Rösiger und Sarah Schulz (2020). „Annotation als flexibel einsetzbare Methode“. In: Reflektierte Algorithmische Textanalyse. Hrsg. von Nils Reiter, Axel Pichler und Jonas Kuhn. Berlin: De Gruyter, S. 125-141.

Willand, Marcus, Evelyn Gius und Nils Reiter (2020). „SANTA: Idee und Durchführung“. In: Reflektierte Algorithmische Textanalyse. Hrsg. von Nils Reiter, Axel Pichler und Jonas Kuhn. Berlin: De Gruyter, S. 391-422. 
